\title{
Studies on the Mechanism of RNAi-dependent Heterochromatin Assembly
}

\author{
D. Moazed, M. Bühler, S.M. Buker, S.U. Colmenares, E.L. Gerace, S.A. Gerber, ${ }^{\dagger}$ \\ E.-J.E. Hong, M.R. Motamedi, A. Verdel, * J. Villén, AND S.P. Gygi \\ Department of Cell Biology, Harvard Medical School, Boston, Massachusetts 02115
}

\begin{abstract}
Assembly of heterochromatin at centromeric DNA regions in the fission yeast Schizosaccharomyces pombe involves an intimate interplay between chromatin modifying complexes and components of the RNAi pathway. The RNA-induced transcriptional silencing (RITS) complex, containing Chp1, Ago1, Tas3, and centromeric siRNAs, localizes to centromeric DNA repeats and is required for the assembly and maintenance of heterochromatin. RITS brings together two types of molecular recognition modules: a chromodomain protein, which binds to lysine 9 methylated histone H3 (H3K9), and Argonaute, which binds to specific sequences by siRNA-directed base-pairing interactions. The RNA-directed RNA polymerase complex (RDRC), composed of Rdp1, the Hrr1 helicase, and the Cid12 Poly(A) polymerase family member, synthesizes doublestranded RNA and creates the substrate for Dicer to generate siRNAs. RDRC physically associates with RITS, and both complexes localize to noncoding centromeric RNAs and centromeric DNA repeats, suggesting that recognition of nascent RNA transcripts may be involved in localization of these complexes to specific chromosome regions. In support of this possibility, tethering of the RITS complex to the transcript of the normally euchromatic $u r a 4^{+}$gene results in siRNA generation and RNAi- and heterochromatin-dependent silencing of the $\mathrm{ura}^{+}$gene. Finally, silencing of a subset of endogenous and transgene promoters within heterochromatic DNA domains occurs by RNAi-dependent degradation of nascent transcripts by a mechanism that we have termed co-transcriptional gene silencing (CTGS).
\end{abstract}

Eukaryotic chromosomes contain two distinct types of chromatin domains, euchromatin and heterochromatin (Heitz 1928). Euchromatin has a decondensed appearance during interphase and defines the gene-rich and presumably transcriptionally active portion of the genome. In contrast, heterochromatin retains the condensed appearance of mitotic chromosomes during interphase and until recently was thought to represent an inaccessible transcriptionally inert state. Heterochromatin is often associated with repetitive DNA sequences and with regions near telomeres or surrounding centromeres, and plays a central role in gene regulation and maintenance of chromosome stability in organisms ranging from yeast to human (Richards and Elgin 2002; Grewal and Moazed 2003). Mutations that perturb heterochromatin assembly therefore result in defects in the recruitment of cohesin to centromeric region and cause chromosome mis-segregation. In addition, other repetitive DNA sequences, transposons, and retroelements, which form up to half of the genomes of many multicellular eukaryotes, are often associated with and stabilized by heterochromatin (Henikoff 1998; Martienssen et al. 2004). Heterochromatin-like structures also control the expression of master developmental regulatory genes and may provide a basis for epigenetic memory mechanisms that maintain cell identity (Paro 1993; Francis and Kingston 2001).

Studies over the past two decades have identified many of the structural components of heterochromatin and have

Present addresses: *Laboratoire de Biologie Moleculaire et Cellulaire de la Differenciation, INSERM U309, Institut Albert Bonniot, Faculte de Medecine, Domaine de la Merci, La Tronche, France; †'DartmouthHitchcock Medical Center, Rubin Building, Lebanon, New Hampshire 03756 . defined how these domains differ from euchromatin at the molecular level (Moazed 2001). The basic unit of chromatin is the nucleosome, which is composed of 147 base pairs of DNA wrapped about two times around an octamer of the four basic histones, H2A, H2B, H3, and H4 (Luger et al. 1997; Kornberg and Lorch 1999). The amino termini of histones play a central role in gene regulation and contain many posttranslational modifications, including acetylation and methylation of specific lysine residues (Grunstein 1997a; Jenuwein and Allis 2001), which regulate the binding of non-histone structural proteins to the nucleosome. In general, heterochromatin and euchromatin are marked with different types of modifications and nonhistone structural proteins. For example, heterochromatic domains contain hypoacetylated histones and methylation on lysine 9 of the amino terminus of histone H3 (H3K9), whereas euchromatic domains have hyperacetylated histone tails and contain methylation on lysine 4 of $\mathrm{H} 3$ (H3K4). Importantly, the enzymes that execute histone deacetylation and H3K9 methylation, together with the proteins that bind the modified histone tails, are structural components of heterochromatin (Grunstein 1997b; Moazed 2001). This observation has given rise to models for how large DNA domains are assembled into heterochromatin by spreading of heterochromatin proteins over large DNA domains from defined nucleation sites (Grewal and Moazed 2003; Rusche et al. 2003; Moazed et al. 2004). In these models, the recruitment of enzymes that modify histone tails creates binding sites for histone-binding proteins that recognize the modified histone tail. Sequential cycles of binding and modification then drive the spreading of heterochromatin along the chromatin fiber until barrier elements prevent further spreading. 
A key structural component of heterochromatin is the enzyme that methylates H3K9 (Rea et al. 2000; Bannister et al. 2001; Lachner et al. 2001; Nakayama et al. 2001). This enzyme is called Su(var)3-9 in Drosophila, Suv39h in human, and $\mathrm{Clr} 4$ in fission yeast. The methylation of H3K9 by Suv39h creates a binding site for the HP1 chromodomain protein (Bannister et al. 2001; Lachner et al. 2001). Two HP1 homologs, called Swi6 and Chp2, together with $\mathrm{Clr} 4$ and several other proteins, are required for heterochromatin formation in fission yeast (Ekwall et al. 1996; Partridge et al. 2000; Thon and Verhein-Hansen 2000). A third fission yeast chromodomain protein, Chp1, is also required for heterochromatin formation, and like Swi6/HP1, recognizes methylated H3K9 and associates with heterochromatic DNA domains (Partridge et al. 2000; Thon and Verhein-Hansen 2000). Heterochromatin assembly also requires several histone deacetylases (HDACs), which deacetylate H3K9 and other sites to help initiate heterochromatin assembly (Grewal 2000).

In addition to histone-binding proteins and modifying enzymes, components of the RNA interference (RNAi) and other RNA silencing pathways, first identified by their role in posttranscriptional gene silencing and RNA-directed DNA methylation in plants, are required for heterochromatin assembly in fission yeast (Volpe et al. 2002). The introduction of double-stranded RNA (dsRNA) into plant cells by transgene-containing retroviruses results in the methylation of homologous DNA sequences and transcriptional gene silencing (TGS) (Wassenegger et al. 1994). Two other silencing pathways are also triggered by dsRNA and share components with the RNA-directed DNA methylation pathway in plants. First, small RNAs of about 22 nucleotides in size, called microRNAs (miRNAs), are processed from larger hairpin RNA precursors by the RNase III family ribonuclease, Dicer, and mediate the translational inactivation of mRNAs containing complementary sequences at their 3'-untranslated regions (Carrington and Ambros 2003). Second, long dsRNA is processed into small interfering RNAs (siRNA) that target complementary mRNAs for degradation (Fire et al. 1998; Hannon 2002). In plants, fungi, and Caenorhabditis elegans, the siRNA silencing pathway requires an RNA-directed RNA polymerase (RdRP) that is thought to synthesize dsRNA and amplify siRNAs (Baulcombe 2004). The primary function of RdRPs may be the recognition of endogenous aberrant RNAs that are produced from transcription of repetitive sequences. Both miRNAs and siRNAs load onto a conserved protein, called Argonaute, which forms the core subunit of the RNA-induced silencing complex (RISC), and uses the small RNAs as guides to find complementary sequences (Hammond et al. 2000; Hutvagner and Zamore 2002; Baulcombe 2004). Argonaute proteins are characterized by conserved PAZ and PIWI domains. The PAZ domain binds siRNA, whereas the RNase H-like PIWI domain directly cleaves target mRNAs (Lingel et al. 2003; Song et al. 2003; Yan et al. 2003; Meister et al. 2004).

The first indication that components of these RNA silencing pathways function in assembly of silent or heterochromatin-like domains came from studies of gene silencing in Drosophila. Birchler and colleagues discovered that repeat-induced gene silencing in Drosophila requires components of the Polycomb group silencing proteins (Pal-Bhadra et al. 2002). The founding member of this family, Polycomb $(\mathrm{Pc})$, is a chromodomain protein that is similar to HP1/Swi6 and participates in the inactivation of homeotic selector genes (Paro 1993). Interestingly, in addition to Polycomb group proteins, this repeat-induced gene silencing requires an Argonaute family protein, called Piwi, and is associated with the production of siRNAs from the repeated locus (Pal-Bhadra et al. 2002). More recently, components of the RNAi pathway have also been identified with roles in heterochromatic gene silencing and position-effect variegation in Drosophila (Pal-Bhadra et al. 2004). Thus, similar to RNA-directed DNA methylation in plants, components of the RNAi pathway are associated with nuclear gene silencing in Drosophila. Another striking example of the role of RNA silencing in genomic reprogramming comes from studies of the massive DNA elimination in the somatic macronucleus of Tetrahymena, which requires components of both chromatin and the RNAi machinery and correlates with siRNA generation (Mochizuki et al. 2002).

RNA silencing proteins, such as Dicer, Argonaute, and RdRP, are conserved in fission yeast, which contains a single gene that codes for each protein $\left(\mathrm{dcrl}^{+}, \mathrm{agol}^{+}\right.$, and $r d p 1^{+}$, respectively). Deletion of any one of these genes results in loss of centromeric heterochromatin in fission yeast (Volpe et al. 2002). Moreover, fission yeast cells contain siRNAs that match the sequence of repetitive DNA elements at centromeres, where heterochromatin assembly is initiated (Fig. 1) (Reinhart and Bartel 2002). Consistent with these observations, fission yeast centromeric repeats are transcribed in both the forward and reverse directions, suggesting a source for dsRNA that can be processed into siRNA (Fig. 1) (Volpe et al. 2002). These observations indicated a conserved relationship between RNA silencing and assembly of repressive chromatin domains in fission yeast, but the basis for this relationship was not understood.

To define the complexes and molecular interactions that assemble and propagate heterochromatin, we have begun a biochemical analysis of the proteins that are required for heterochromatin formation in fission yeast. Our studies have identified several complexes that collaborate to initiate and propagate epigenetic chromatin domains in an RNAi- and heterochromatin-dependent manner and have established a direct role for small RNAs in heterochromatin assembly. In addition, our studies have provided insight into how small RNAs target specific chromosome regions and have uncovered an unanticipated silencing mechanism that appears to involve the co-transcriptional degradation of transcripts in heterochromatin. In this paper, we review the fission yeast RNAi and heterochromatin complexes and discuss experiments that provide insight into their mechanism of action.

\section{RITS, AN SIRNA-CONTAINING COMPLEX THAT PHYSICALLY LINKS RNAI TO HETEROCHROMATIN}

We began our biochemical purifications by focusing on chromodomain proteins. Purification of one such protein, Chp1, resulted in the identification of a ribonucleo- 


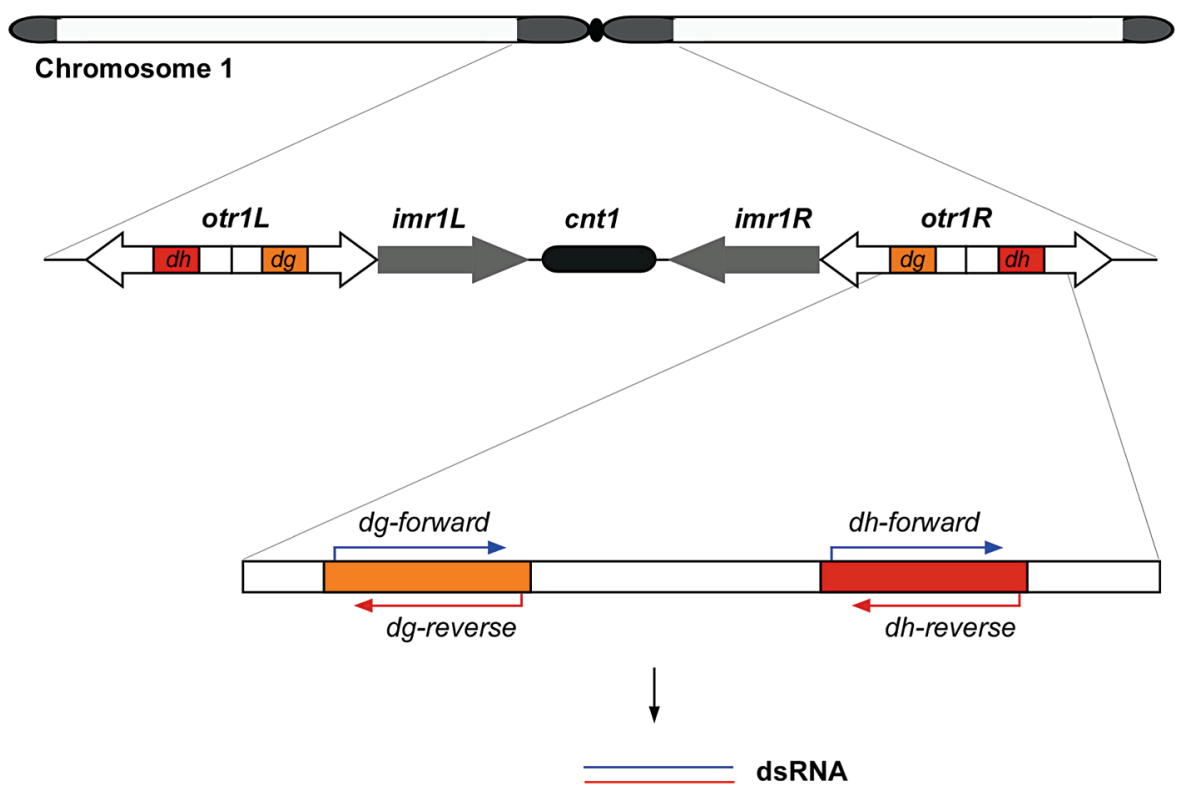

Figure 1. Schematic diagram of S. pombe chromosome 1. Regions surrounding the centromere and telomeres, which are packaged into heterochromatin, are highlighted in dark gray. The central core region (cnt), composed of unique sequences, is flanked by innermost repeats $(\mathrm{imr}$ ) and outermost repeats (otr). The otr regions of chromosome 1 contain a single copy of each $d g$ and $d h$ repeats, which are transcribed in both directions. These forward and reverse transcripts are a source of centromeric siRNA, although it is unclear whether they base-pair directly to form dsRNA.

protein complex called the RITS (RNA-induced transcriptional silencing) complex (Verdel et al. 2004). In addition to Chp1, RITS contains the fission yeast Argonaute protein, Ago1, Tas3, and approximately 22to 25-nucleotide centromeric siRNAs (Fig. 2). RITS therefore provides a direct physical link between small RNAs, an RNAi protein, and a heterochromatin protein. Like Ago1 and Chp1, the Tas3 subunit of RITS is required for heterochromatin assembly at centromeres. Moreover, the localization of RITS to centromeric chromatin is siRNA-dependent, because in $\operatorname{dcr} 1 \Delta$ cells, which lack centromeric siRNA, the structural integrity of RITS is maintained, but the complex devoid of siRNA no longer cross-links to centromeric repeats (Verdel et al. 2004). This suggests that siRNAs act as guides that target the complex to specific chromosome regions by basepairing interactions (Fig. 3).

An important feature of the RITS complex is that it brings together two different types of molecular recognition modules, one involving base-pairing interactions and the other involving histone tail binding. First, the Agol protein binds to siRNAs, which act as guides for association with target nucleic acid sequences in RITS and the related RISC (Verdel et al. 2004). Second, the Chp1 subunit of RITS contains an amino-terminal chromodomain that specifically binds to methylated $\mathrm{H} 3 \mathrm{~K} 9$ and localizes throughout heterochromatic DNA domains (Partridge et al. 2000). The Tas 3 subunit is of unknown function but contains a central approximately 130-amino acid domain with several glycine-tryptophan repeats (Fig. 2) (referred to as GW repeats; Eystathioy et al. 2002). Interestingly, these repeats are also present in the human and Drosophila GW182 protein, which has recently been shown to interact with Ago1 and target it to nuclear RNA processing bodies, called the P-bodies (Behm-Ansmant et al. 2006). The stable association of RITS with its target chromosome regions at centromeres appears to require interactions that involve siRNAs as well as methylated H3K9. For example, the association of Chp1 and other subunits of RITS with centromeric DNA regions is greatly diminished in clr4 mutant cells, which lack H3K9 methylation (Partridge et al. 2002; Noma et al. 2004). On the basis of these observations, Verdel et al. (2004) proposed that RITS associates with target chromosome regions via base-pairing interactions and recruits the $\mathrm{Clr} 4$ H3K9 methyltransferase to initiate heterochromatin formation. Initial H3K9 methylation is hypothesized to stabilize the complex on chromatin and to promote the recruitment of additional RNAi complexes and the spreading of heterochromatin (Fig. 3).

In principle, siRNAs can guide the RITS complex to specific chromosome regions via base-pairing with either an unwound DNA double helix or nascent RNA transcripts (Grewal and Moazed 2003; Verdel and Moazed 2005). There is precedent for both types of recognition mechanisms. RNA-DNA hybrids occur during the recognition of single-stranded chromosome ends by the RNA component of telomerase, which acts as a guide that helps localize telomerase to the chromosome end (Blackburn 1991). Examples of siRNA-RNA base-pairing are welldocumented for the RISC comlex (Hannon 2002), and RNA-RNA base-pairing interactions that guide specific molecular recognition events also occur during protein synthesis and numerous RNA processing events, including pre-mRNA splicing and snoRNA-mediated rRNA methylation (Huttenhofer and Schattner 2006). The biochemical and in vivo experiments described below provide strong support for siRNA-RNA interactions during 


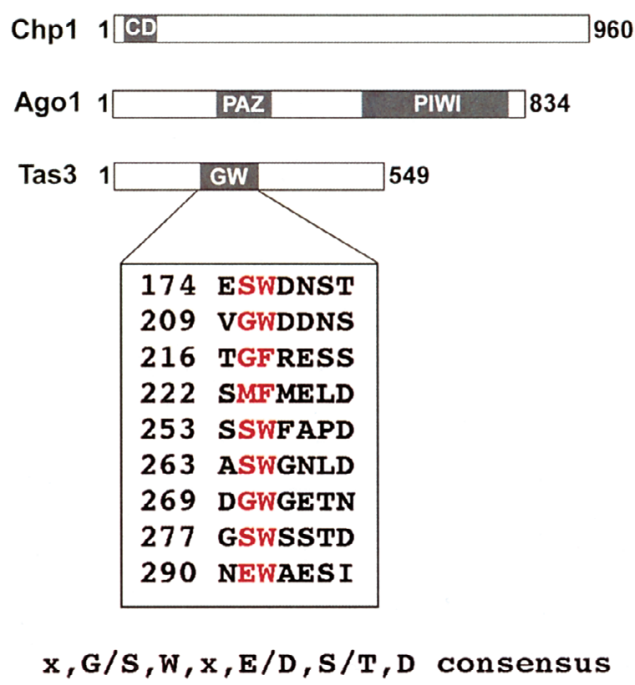

Figure 2. Structural organization of the subunits of the RITS complex. (A) The Chp1 protein contains an amino-terminal chromodomain that associates with methylated histone H3K9. The Argonaute family protein, Ago1, contains typical PAZ and PIWI domains that bind to siRNA and cleave target RNAs, respectively. $(B)$ The Tas 3 protein contains a central GW repeat domain, which has been found in Argonaute- and RNA-binding proteins in multicellular eukaryotes. (Modified, with permission, from Verdel et al. 2004 [C AAAS].)

RNAi-mediated heterochromatin assembly; nonetheless, siRNA-DNA base-pairing cannot be ruled out, as the two mechanisms are not mutually exclusive.

\section{RDRC AND ITS ASSOCIATION WITH RITS AND CENTROMERIC RNA TRANSCRIPTS}

RdRPs are involved in dsRNA synthesis during RNAi (Baulcombe 2004). How these enzymes select their RNA substrates to initiate dsRNA synthesis is not understood. The fission yeast RdRP, Rdp1, is physically associated with centromeric DNA repeats and is required for heterochromatin assembly, suggesting that it plays a direct role in this process (Volpe et al. 2002; Sugiyama et al. 2005). Purification of Rdp1 shows that it is associated with two other proteins, Cid12 and Hrr1, in a complex termed RDRC (Fig. 4) (Motamedi et al. 2004). Like Rdp1 itself, Hrr1 and Cid12 are required for RNAi and heterochromatin assembly (Motamedi et al. 2004). The Cid12 protein is a member of the $\beta$-family of nucleotidyl transferases. This family includes the canonical poly(A) polymerase (PAP) and the mammalian oligoadenylate synthetase (OAS), which as part of the interferon response modifies dsRNA with $2^{\prime}-5^{\prime}$ linked oligo(A) tails to activate RNA degradation (Fig. 4) (Justesen et al. 2000; Saitoh et al. 2002). Several members of this family have also been implicated in regulation of RNA metabolism. The Saccharomyces cerevisiae homologs, Trf4 and Trf5, are involved in a nuclear surveillance mechanism that targets aberrant RNAs, lacking proper processing signals, for degradation by the nuclear exosome complex (LaCava et al. 2005; Vanacova et al. 2005). Purified Trf4, as well as Cid1 and Cid13, two

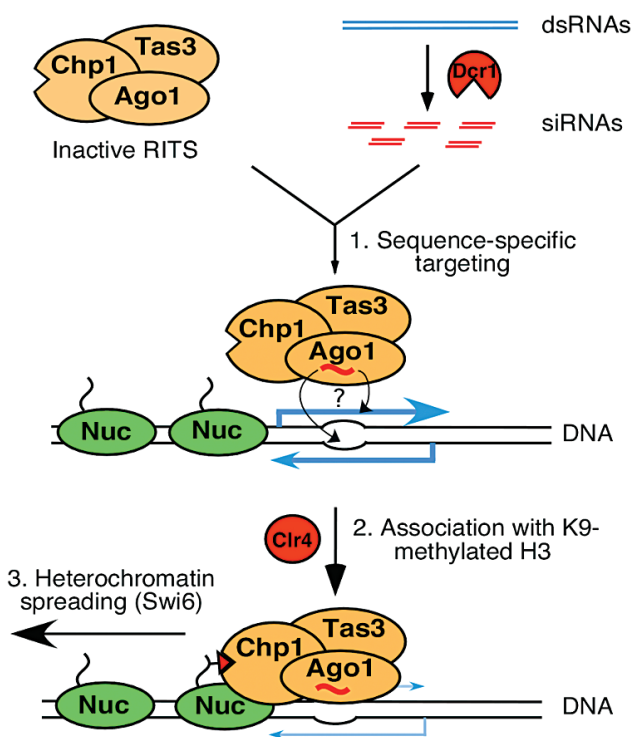

Figure 3. The RITS complex composed of Chp1, Ago1, Tas3, and siRNA targets specific chromosome regions for assembly into heterochromatin. The stable association of RITS with centromeric repeats requires both siRNAs and H3K9 methylation. (Adapted, with permission, from Verdel et al. 2004 [C AAAS].)

cytoplasmic fission yeast family members, have poly(A) polymerase activity in vitro, which is thought to be involved in targeting of transcripts for degradations (Read et al. 2002; Saitoh et al. 2002; Haracska et al. 2005).

The precise role of Cid12 in RNAi-mediated gene silencing is unknown. The presence of Cid12 in the RDRC suggests possible parallels between the role of Cid12-like proteins, such as Trf4 and Trf5, in degradation of aberrant euchromatic transcripts and the targeting of centromeric RNAs for conversion into dsRNA. Cid12 may help target aberrant centromeric transcripts to RDRC rather than the exosome. In addition to being processed into siRNA, the cleavage of RDRC-synthesized dsRNA by Dicer may be part of the mechanism that degrades heterochromatic transcripts. Alternatively, Cid12 may modify centromeric RNAs, either before or after dsRNA synthesis, so that they could be recognized by chromatin modifying complexes that initiate heterochromatin assembly (Motamedi et al. 2004; see below). Cid12-like proteins are highly conserved and function in RNAi silencing in other organisms. Rde3, a member of this family of nucleotidyl transferases, is required for siRNA accumulation and RNAi in C. elegans (Chen et al. 2005).

Hrr1 shares weak sequence similarity to Smg2 and Sde3, which are required for RNAi in C. elegans and Neurospora, respectively (Motamedi et al. 2004). However, closer relatives of Hrr1 are present in other fungi and metazoans, but their possible role in RNAi has not been determined (Fig. 4). It is unclear whether Hrr1 has helicase activity or, alternatively, serves as an ATPase motor that promotes the assembly of RNAi complexes on target RNA molecules.

To determine whether the fission yeast Rdp1 has RNAdirected RNA polymerase activity, we carried out in vitro 
A

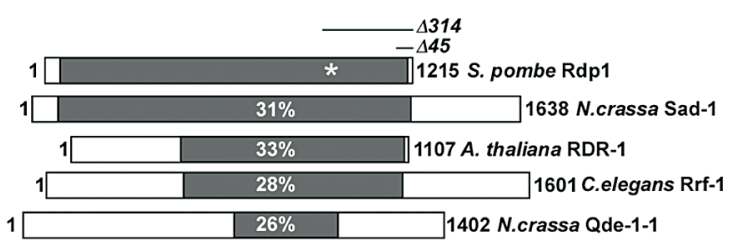

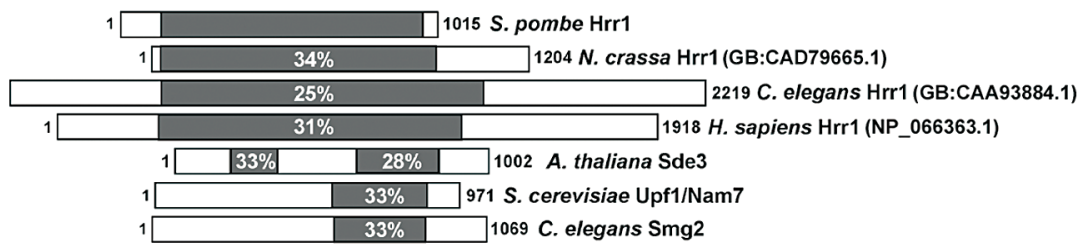

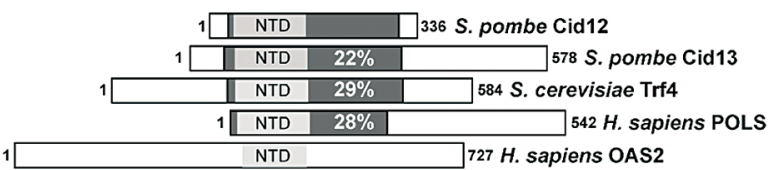

B

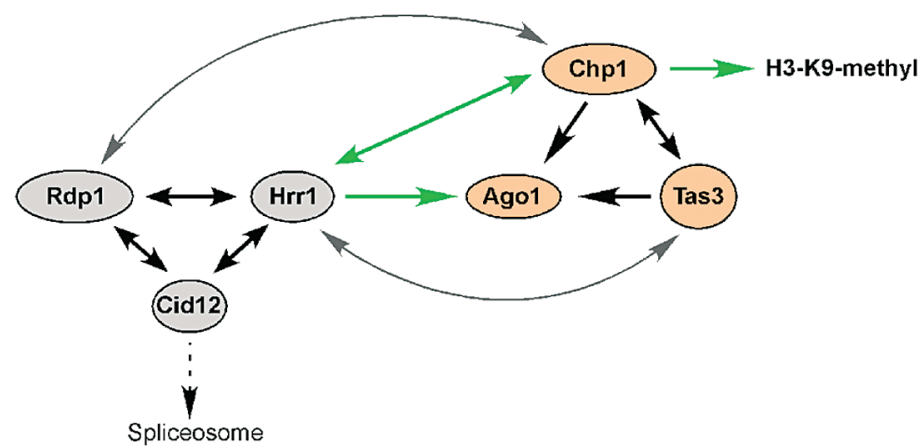

Figure 4. (A) Subunits of the RDRC. Rdp1 shares nucleotidyltransferase domains with other RNA-directed RNA polymerases. Truncations of the carboxy-terminal region of the RdRP domain ( $\Delta 314$ and $\Delta 45)$ or substitution of D903 with A (denoted by asterisk) abolish the in vitro activity of RDRC and in vivo RNAi-dependent heterochromatin assembly. (B) Summary of protein-protein interactions between subunits of the RDRC (Rdp1, Hrr1, Cid12) and RITS (Chp1, Ago1, and Tas3) complexes detected by biochemical purifications. Straight arrows indicate interactions uncovered by mass spectrometry of mixtures of purified proteins; curved arrows indicate interactions based on co-immunoprecipitation assays; green arrows indicate interactions that are Dicer- and Clr4-dependent. (Adapted, with permission, from Motamedi et al. 2004 [C Elsevier].)

reactions to test whether purified RDRC could synthesize dsRNA using a single-stranded RNA template. We found that RDRC has template-directed RNA synthesis activity in vitro (Motamedi et al. 2004). This activity does not require an RNA primer but always produces cRNA products that are the same size as the template, suggesting that the enzyme complex begins RNA synthesis at or near the 3 'end of the template. Furthermore, an amino acid substitution in the conserved catalytic core of the enzyme, or truncations that remove portions of the conserved core, abolish dsRNA synthesis activity in vitro and gene silencing in vivo, suggesting that dsRNA synthesis activity is required for heterochromatin assembly (see Fig. 4) (Motamedi et al. 2004; Sugiyama et al. 2005). The primer-independent activity of
RDRC is similar to that observed for the distantly related Neurospora RdRP, Qde1, and raises questions about how these enzymes recognize specific RNA substrates in vivo (Makeyev and Bamford 2002). One possibility is that RDRC is recruited to appropriate transcripts by an siRNAprogrammed RITS complex. Indeed, RDRC physically interacts with the RITS complex in a Dicer- and Clr4dependent manner, indicating that the association requires siRNAs and/or chromatin interactions. RDRC may therefore associate with RNA templates that are targeted by an siRNA-programmed RITS to begin second-strand synthesis in a primer-independent fashion. Consistent with this hypothesis, RNA-ChIP experiments show that components of both the RITS and RDRC complexes associate with cen- 
tromeric RNA transcripts in a Dicer- and Clr4-dependent manner (Motamedi et al. 2004).

The requirement for $\mathrm{Clr} 4$ in association of RITS and RDRC suggests that this association involves nascent, chromatin-bound, transcripts. To determine whether centromeric RNAs are nuclear and chromatin associated, we examined their distribution in chromatin and cytoplasmic fractions. In contrast to actin mRNA, which is predominantly cytoplasmic, centromeric $d g$ and $d h$ transcripts are exclusively associated with a chromatin pellet fraction, but become distributed equally between chromatin and cytoplasmic fractions in $d c r 1 \Delta$ or $\operatorname{clr} 4 \Delta$ cells (M. Motamedi and D. Moazed, unpubl.). Together with the RNA-ChIP experiments (Motamedi et al. 2004), mentioned above, these observations demonstrate that RNAi complexes are associated with chromatin-bound centromeric RNA transcripts and support a role for the nascent transcript as a platform for the initial localization of RITS and RDRC to specific chromosome regions (Fig. 5).

\section{THE CLRC COMPLEX CONTAINS Clr4, Rik1, Cul4, AND PROTEINS OF UNKNOWN FUNCTION}

The studies described so far provide a possible explanation for how the RNAi machinery is targeted to specific chromosome regions (Figs. 2 and 5), but it remains unclear

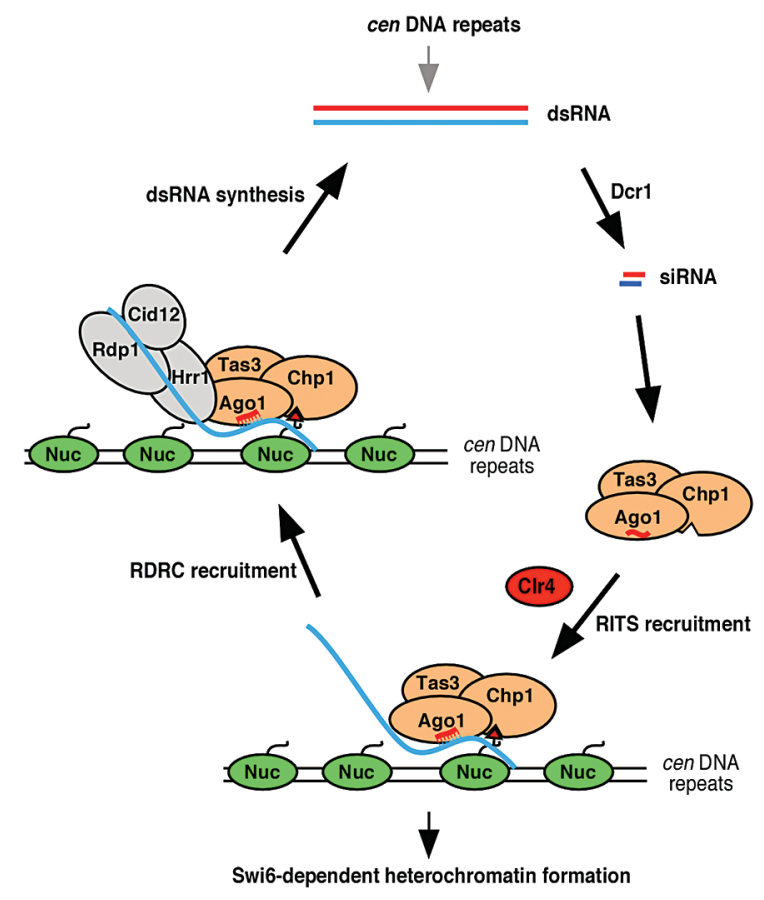

Figure 5. Targeting of the nascent transcript by an siRNA-programmed RITS complex and a model for chromosome-associated dsRNA synthesis and siRNA generation. siRNA-programmed RITS associates with nascent pre-mRNA via base-pairing interactions. The recruitment of Clr4 and methylation of $\mathrm{H} 3 \mathrm{~K} 9$ then allow the stable association of RITS with chromatin and the recruitment of RDRC. dsRNA synthesis by RDRC and its processing by Dicer generate additional siRNAs, which target new RITS complex to the chromosome and create a positive feedback loop that helps propagate heterochromatin. (Adapted, with permission, from Motamedi et al. 2004 [C Elsevier].) how RITS and/or RDRC recruits histone modifying activities such as deacetylases and the histone H3K9 methyltransferase to chromatin. To understand how RNAi may recruit the chromatin modification machinery, we have been purifying the histone deacetylase and methyltransferase enzymes. We initially focused on the Clr4 H3K9 methyltransferase, which had previously been shown to coimmunoprecipitate with Rik1 (Sadaie et al. 2004). Rik1 is associated with heterochromatin and is required for heterochromatin formation, but its function is unknown. We chose to purify Rik1 because, unlike Clr4, cells carrying an affinity-tagged version of Rik1 were phenotypically wild type for silencing. Purification of Rik1 shows that, in addition to Clr4, it is associated with the Cullin 4 (Cul4) E3 ubiquitin ligase and two proteins of unknown function, Cmc1 (also called Raf1, Dos1, and Clr8) and Cmc2 (also called Raf2, Dos2, and Clr7) in a complex that we have named CLRC (Clr4-Rik1-Cul4)(Hong et al. 2005; Horn et al. 2005; Jia et al. 2005; Li et al. 2005; Thon et al. 2005). The CLRC complex is architecturally similar to other Cullin ubiquitin ligase complexes, but its composition does not provide a ready answer for how it is recruited to chromatin by the RNAi pathway (Fig. 6). A possible clue comes from the sequence similarity of Rik1 to nucleic acid binding proteins. Rik1 is a beta propeller WD repeat protein that belongs to a subfamily of WD repeat proteins that includes the Ddb1 DNA damage protein, the CPSF-A subunit of the poly(A) polymerase complex, and some splicing factors (Fig. 6) (Hong et al. 2005). The Ddb1 and CPSF-A proteins are involved in the recognition of damaged DNA and polyadenylation signals, respectively (Barabino et al. 2000; Hu et al. 2004). An intriguing possibility is that Rik1 recognizes a nucleic acid species that is generated during the initial steps in RNAi targeting of the genome; for example, the siRNA-nascent pre-mRNA duplex, or the dsRNA generated by the RDRC, or perhaps a poly(A) tail generated by Cid12 (Motamedi et al. 2004; Hong et al. 2005). Alternatively, the CLRC complex may be directly recruited to chromatin through physical interactions with one or more subunits of the RITS or RDRC complexes, but these interactions may not be stable enough to withstand biochemical isolation. The role of the Cul4 E3 ubiquitin ligase remains to be determined, but its association with CLRC and requirement for heterochromatin formation suggest that ubiquitination of one or more proteins is required for heterochromatin assembly.

Recent evidence indicates that some $\mathrm{H} 3 \mathrm{~K} 9$ methylation at centromeric DNA repeats occurs independently of RNAi. In RNAi mutant cells, H3K9 methylation at cen repeats is reduced but not abolished ( $\mathrm{Li}$ et al. 2005; Yamada et al. 2005). This methylation requires Clr4 and its associated partners in the CLRC complex, suggesting that the complex can be recruited to centromeres in an RNAi-independent fashion. These observations provide a possible explanation for the role of Clr4 in association of RITS with chromatin and suggest that low levels of H3K9 methylation present at centromeres may help recruit RITS via the chromodomain of its Chp1 subunit. However, efficient recruitment to centromeres and the spreading of H3K9 methylation require an siRNA programmed RITS. For example, in the absence of RNAi, reporter genes that 
A

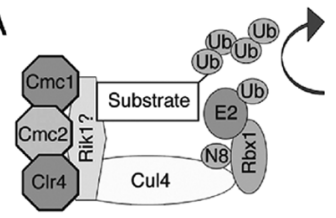

B

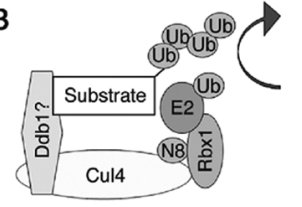

C

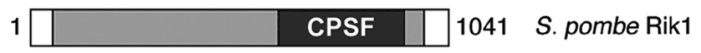

\section{1} CPSF 1072 S. pombe Ddb1

Figure 6. Composition of the Clr4-Rik1-Cul4 (CLRC) complex and its overall similarity to Cul4 ubiquitin ligase complexes that have been implicated in DNA damage repair. In Cul4-Ddb1 complexes, damaged DNA triggers downstream ubiquitination events that are required for repair. By analogy, dsRNA or other siRNA-dependent structures may activate CLRC and promote heterochromatin assembly. (Adapted, with permission, from Hong et al. 2005 [C Landes Bioscience].)

are inserted in centromeric repeats are active and lack H3K9 methylation, indicating that RNAi is required for the spreading of $\mathrm{H} 3 \mathrm{~K} 9$ methylation into the reporter gene (Volpe et al. 2002), as well as maximal H3K9 methylation at centromeric repeats.

\section{MECHANISM OF SIRNA GENERATION}

Centromeric DNA repeats in Schizosaccharomyces pombe are transcribed in both the forward and reverse directions, generating overlapping transcripts that can potentially base-pair to produce dsRNA (Fig. 1). This dsRNA would be expected to act as a substrate for processing by Dicer to generate siRNA. It was therefore surprising to find that the requirements for siRNA generation extend far beyond Dicer (Motamedi et al. 2004; Noma et al. 2004). In addition to Dicer, the generation of siRNAs absolutely requires components of the RDRC, RITS, and CLRC complexes (Motamedi et al. 2004; Noma et al. 2004; Hong et al. 2005; Li et al. 2005). Moreover, siRNA levels are reduced in sir2 and swi6 mutant cells (Motamedi et al. 2004; Bühler et al. 2006). These results clearly demonstrate that the biogenesis of heterochromatic siRNAs is much more complex than the simple processing of dsRNA generated by overlapping forward and reverse transcripts. On the basis of the biochemical studies described above, we have proposed that dsRNA synthesis and siRNA generation occur on the chromosome and require the recruitment of the RDRC complex to chromatin-associated centromeric transcripts by the RITS complex (Fig. 5) (Motamedi et al. 2004).

The source of the initial trigger siRNAs remains speculative. It is possible that a small amount of siRNA is in fact produced independently of RDRC activity and begins the positive feedback loop depicted in Figure 5. If this is the case, these trigger siRNAs are below our current detection limits. An alternative possibility is that the recognition of a specific structural feature of centromeric $d g$ and $d h$ transcripts by RDRC is required to initiate the dsRNA synthesis and siRNA amplification loop.
Regardless of the mechanism, a remarkable conclusion of the above studies is that dsRNA synthesis and siRNA generation are chromatin-coupled events. This coupling may be a unique feature of chromosome-associated RNAi that distinguishes it from RNAi-mediated posttranscriptional gene silencing.

\section{TETHERING RITS TO NASCENT TRANSCRIPTS INITIATES HETEROCHROMATIN FORMATION}

The siRNA-nascent transcript model proposes that the initial localization of RITS to the nascent transcript via base-pairing interactions triggers the downstream events that initiate heterochromatin assembly. We sought to test this model by investigating whether the tethering of RITS to the RNA transcript of a normally active euchromatic gene could initiate heterochromatin assembly. We fused the Tas 3 subunit of RITS to $\lambda \mathrm{N}$, a phage lambda site-specific RNA-binding protein, to generate Tas $3-\lambda \mathrm{N}$ and asked whether Tas $3-\lambda \mathrm{N}$ could silence the expression of a $\mathrm{ura}^{+}$gene, modified to express a transcript that contained five $\lambda \mathrm{N}$-binding sites at its $3^{\prime}$-untranslated region (ura $4^{+}-5$ BoxB) (Fig. 7) (Bühler et al. 2006). We found that this tethering results in silencing of $\mathrm{urat}^{+}-5 B$ oxB with a similar efficiency as silencing of a $\mathrm{urat}^{+}$reporter gene inserted within the centromeric repeat regions (Bühler et al. 2006). Tethered silencing depends on components of the RNAi pathway as well as heterochromatin proteins Clr4, Swi6, and Sir2, thus displaying genetic requirements that are similar to silencing at centromeres. Furthermore, Tas $3-\lambda \mathrm{N}$ silencing is accompanied by the generation of $\mathrm{ura}^{+}$siRNAs. These results demonstrate that tethering RNAi components to a nascent RNA transcript could initiate chromatin modifications and, furthermore, show that siRNAs can be generated in a primer-independent manner in vivo (Bühler et al. 2006). The latter observation is consistent with earlier findings that in vitro the RDRC complex synthesizes dsRNA in a primer-independent manner (Motamedi et al. 2004).

The tethered silencing system and the observation that it is accompanied by $u \mathrm{rat}^{+}$siRNA generation provided the opportunity to ask whether the newly generated siRNAs could act in trans to silence a second copy of $\mathrm{ura}^{+}$. Surprisingly, although $\mathrm{ura}^{+}$siRNAs load onto the RITS complex, they are incapable of silencing a second copy of $\mathrm{ura4}^{+}$in trans (Bühler et al. 2006). This cis-

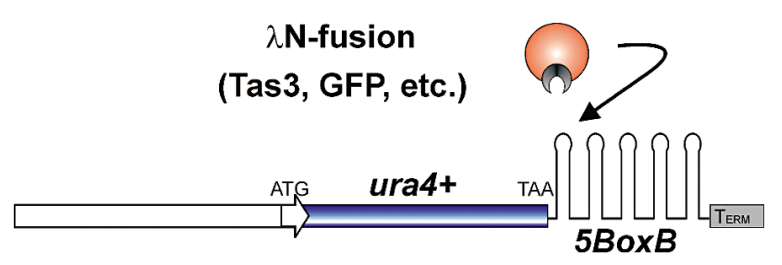

Figure 7. Strategy for targeting components of the RNAi pathway to $\mathrm{ura}^{+} \mathrm{RNA}$ by fusion to the phage lambda site-specific RNA protein $(\lambda N)$. $\lambda \mathrm{N}$ is a 30 -amino acid domain that binds tightly to an RNA stem-loop structure, called BoxB. Five BoxB stem-loops were inserted at the $3^{\prime}$-untranslated region of $\mathrm{ura}^{+}$. (Adapted, with permission, from Bühler et al. 2006 [C Elsevier].) 
restriction has important implications for the mechanism of siRNA-mediated heterochromatin assembly. First, it indicates a fundamental difference between siRNA-mediated chromatin modification mechanisms and siRNAand micoRNA-dependent PTGS. PTGS mechanisms involve the action of small RNAs, associated with RISC complexes, on cytoplasmic pools of target mRNA (Plasterk and Ketting 2000; Bartel 2004; Filipowicz et al. 2005), and cannot in any way distinguish between mRNA products of different alleles. cis-Restriction, on the other hand, allows identical alleles of the same gene to exist in different epigenetic states, on or off, within the same cell. Second, cis-restriction suggests that siRNAs produced from the $u r a 4^{+}-5 B o x B$ transcript are produced at the site of transcription on nascent RNA transcripts. Otherwise, it is difficult to imagine how the resulting $\mathrm{ura}^{+}{ }^{+}$siRNAs are able to distinguish between the two $\mathrm{ura}^{+}$alleles (Fig. 8).

siRNAs generated on chromatin at the site of dsRNA synthesis may be cis-restricted because their range of action may be limited by a threshold concentration required for siRNA-directed initiation events. In this regard, the RNAi response has been shown to be negatively regulated by Eri1, an exoribonuclease that downregulates siRNA levels in $C$. elegans (Kennedy et al. 2004). Eril is highly conserved and also negatively regulates siRNA levels and heterochromatin formation in fission yeast (Iida et al. 2006). Furthermore, Eri1 is responsible for the $c i s$-restriction of Tas $3-\lambda \mathrm{N}$ silencing as deletion of $\mathrm{eril}^{+}$results in weak Tas $3-\lambda \mathrm{N}$-dependent silencing of a second $\mathrm{ura}^{+}$allele inserted on a different chromosome (Fig. 8) (Bühler et al. 2006). This observation indicates that $S$. pombe siRNAs are able to initiate de novo heterochromatin assembly, but this ability is under strong negative control that is mediated at least in part by siRNA degradation. Eri1-mediated siRNA degradation is likely to play a general role in preventing siRNA levels from going beyond the threshold level required for trans silencing and may prevent spurious siRNA-mediated silencing of the genome.

Although siRNAs can initiate heterochromatin assembly, this event appears to occur at a low frequency even in eril $1 \Delta$ cells (Bühler et al. 2006), suggesting that other factors may limit siRNA-directed chromatin modifications or may be required for the initiation event together with siRNAs. One possibility is that the Clr4 H3K9 methyltransferase, which can be recruited to chromatin independently of RNAi (Noma et al. 2004; Li et al. 2005), provides an initial methyl $\mathrm{H} 3 \mathrm{~K} 9$ mark that increases the efficiency of RITS association with its target chromosome regions.

\section{CTGS AS A PRIMARY MECHANISM OF SILENCING IN HETEROCHROMATIC DNA REGIONS}

Heterochromatin is generally viewed as the inaccessible and transcriptionally inert portion of the genome. This view is partly based on early cytological evidence, which defined heterochromatin as chromosome regions that retain the condensed appearance of mitotic chromosomes throughout the cell cycle (Heitz 1928; Richards and Elgin 2002). However, not all evidence is consistent with this view. For example, heterochromatic domains in S. cerevisiae are accessible to modification by exogenously introduced dam methyltransferase and do not inhibit the binding of RNA polymerase II (RNApII) transcription
A
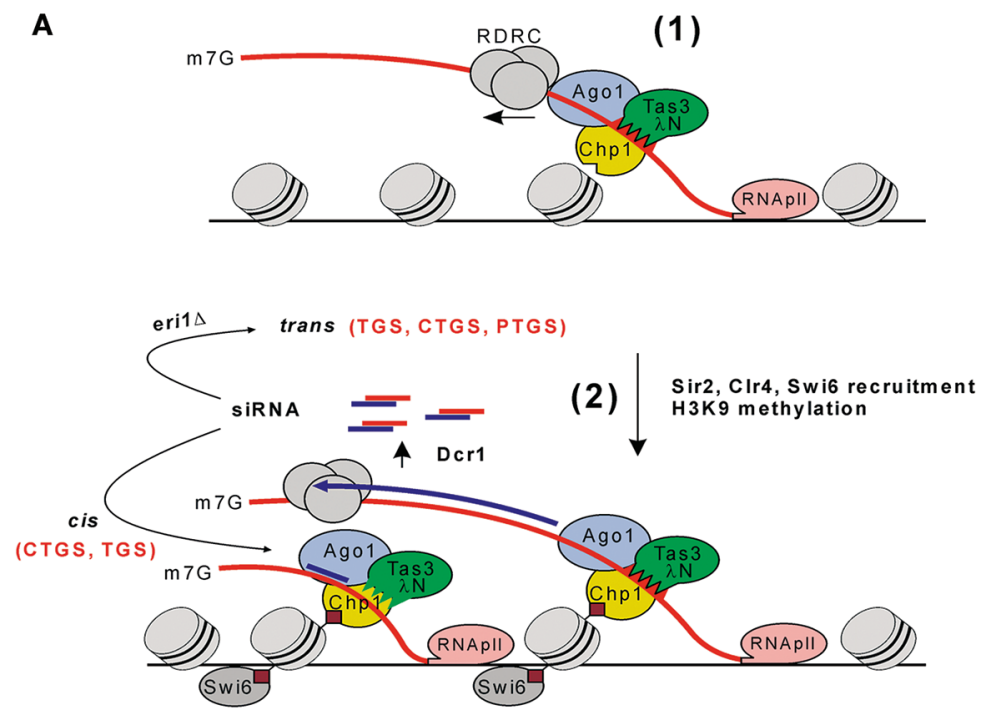

B

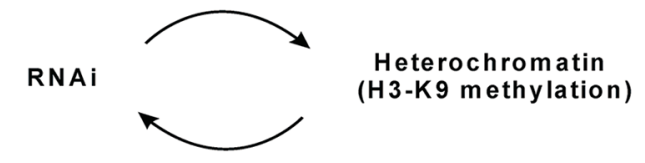

Figure 8. Model for RNAi- and heterochromatin-mediated gene silencing. (A) Tethering of the Tas3 subunit of RITS to an RNA transcript via the $\lambda \mathrm{N}-5 \mathrm{~B}$ oxB system mimics the association of RITS with a nascent transcript through siRNA-dependent base-pairing interactions and initiates dsRNA synthesis, siRNA generation, and silencing of the gene. Tethering of RITS mediates the degradation of the nascent transcript (CTGS) and initiates chromatin modifications, which can also mediate TGS. siRNA-mediated silencing is $c i s$-restricted in wild-type cells, but the deletion of $\mathrm{eril}^{+}$, a conserved exoribonuclease, allows siRNAs to act in trans. (B) siRNAmediated trans-silencing in erils cells demonstrates that RNAi can initiate de novo heterochromatin assembly. Conversely, the ability of RITS to bind to methylated H3K9 and to initiate primer-independent dsRNA synthesis by RDRC suggests that heterochromatin may be able to initiate RNAi to promote the degradation of nascent transcripts. (Adapted, with permission, from Bühler et al. 2006 [C Elsevier].) 
preinitiation complex (Pirrotta and Gross 2005). Similarly, the targeting of Polycomb complexes to a heat shock reporter gene, which results in silencing by a mechanism that resembles heterochromatin formation, does not prevent the association of RNApII preinitiation complex with the promoter of the repressed gene (Breiling et al. 2001; Dellino et al. 2004). Finally, centromeric DNA repeats in $S$. pombe, which are assembled into heterochromatin, give rise to noncoding transcripts that trigger RNAi, indicating that heterochromatic DNA repeats can be transcribed (Volpe et al. 2002).

We were interested in testing whether Tas $3-\lambda N$ silencing, which is accompanied by H3K9 methylation and requires the heterochromatin machinery, involves the inhibition of transcription (Bühler et al. 2006). Transcription nuclear run-on experiments show no change in $u r a 4^{+}-5 B o x B$ transcription rates as a result of silencing in Tas3- $\lambda \mathrm{N}$ cells (Bühler et al. 2006). Furthermore, chromatin immunoprecipitation experiments show that silencing is not accompanied by a reduction in RNApII occupancy across the $u r a 4^{+}-5$ BoxB locus. These observations suggest that although silencing is accompanied by repressive chromatin modifications and requires the heterochromatin machinery, it occurs at a step following transcriptional initiation that is likely to involve RNAi-dependent degradation of nascent transcripts. Because the mechanism is clearly distinct from PTGS, in that it is cis-restricted and occurs on the chromosome, we have proposed the name co-transcriptional gene silencing (CTGS) to distinguish it from classic TGS and PTGS (Bühler et al. 2006). Importantly, CTGS is not specific for tethered silencing. Bühler et al. (2006) also examined the effect of RNAi-mediated heterochromatin assembly at the endogenous $d g$ and $d h$ centromeric repeats as well as at $u \mathrm{ra}^{+}$reporter genes inserted at the otr and imr regions of centromeres (otrlR::ura $4^{+}$and imr $1 R::$ ura $^{+}{ }^{+}$) (see Fig. 1 for map). Transcripts from both the $d g$ and $d h$ repeats and the $u r a 4^{+}$reporter genes inserted within otr and imr accumulate in heterochro-

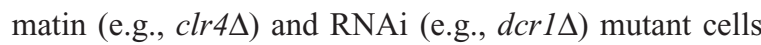
(Bühler et al. 2006). Strikingly, the $\mathrm{ura}^{+}{ }^{+}$reporter genes and the cen- $d h$ region are associated with similar levels of RNApII in wild-type cells and in cells that lack $d c r l^{+}$or $\mathrm{clr} 4^{+}$, indicating that silencing does not inhibit the association of RNApII with these regions. However, loss of heterochromatin does result in a marked increase in RNApII occupancy at the cen- $d g$ repeats, indicating the existence of regional differences in the mechanism of silencing. These results are consistent with previous findings, showing that loss of heterochromatin does not affect the rate of transcription for the cen- $d g$ reverse transcript (Volpe et al. 2002). Together, these studies suggest that RNAi-mediated co-transcriptional degradation of RNA transcripts in heterochromatin (CTGS) is the primary mechanism of silencing for some, but not all, heterochromatic genes.

\section{CONCLUSIONS}

Studies on the mechanism of heterochromatin assembly in fission yeast have uncovered a direct link between the RNAi machinery and chromatin-dependent gene silenc- ing. Key RNA silencing factors such as Argonaute, as well as siRNAs, are associated in complexes with structural components of heterochromatin. Moreover, the biogenesis of dsRNA and siRNA, central players in RNA silencing mechanisms, requires heterochromatin formation and occurs on the chromosome. Furthermore, nascent noncoding centromeric RNAs act as platforms for the siRNAdirected assembly of RNAi and chromatin modifying enzymes, thus revealing a previously unanticipated genetic recognition mechanism. Finally, dissection of the mechanism of RNAi-dependent silencing in heterochromatin indicates that co-transcriptional degradation of nascent transcripts is an important component of gene silencing in these chromosome domains. Like TGS, CTGS involves heterochromatin assembly and the spreading of repressive chromatin modifications such as H3K9 methylation and Swi6/HP1 binding. Unlike TGS, CTGS does not correlate with a reduction in RNApII transcription initiation rates and instead involves the degradation of nascent transcripts. CTGS is distinguished from PTGS in that it requires epigenetic chromatin modifications, is chromosome-associated, and can be regulated in cis.

\section{ACKNOWLEDGMENTS}

The authors thank the National Institutes of Health, the Leukemia and Lymphoma Society, the Carolyn and Peter Lynch Foundation, the Giovannie Armenise-Harvard Foundation, and the Hellman Family Trust Fund for supporting their research.

\section{REFERENCES}

Bannister A.J., Zegerman P., Partridge J.F., Miska E.A., Thomas J.O., Allshire R.C., and Kouzarides T. 2001. Selective recognition of methylated lysine 9 on histone H3 by the HP1 chromo domain. Nature 410: 120.

Barabino S.M., Ohnacker M., and Keller W. 2000. Distinct roles of two Yth $1 \mathrm{p}$ domains in $3^{\prime}$-end cleavage and polyadenylation of yeast pre-mRNAs. EMBO J. 19: 3778 .

Bartel D.P. 2004. MicroRNAs: Genomics, biogenesis, mechanism, and function. Cell 116: 281.

Baulcombe D. 2004. RNA silencing in plants. Nature 431: 356.

Behm-Ansmant I., Rehwinkel J., Doerks T., Stark A., Bork P., and Izaurralde E. 2006. mRNA degradation by miRNAs and GW182 requires both CCR4:NOT deadenylase and DCP1:DCP2 decapping complexes. Genes Dev. 20: 1885.

Blackburn E.H. 1991. Structure and function of telomeres. Nature 350: 569.

Breiling A., Turner B.M., Bianchi M.E., and Orlando V. 2001. General transcription factors bind promoters repressed by Polycomb group proteins. Nature 412: 651.

Bühler M., Verdel A., and Moazed D. 2006. Tethering RITS to a nascent transcript initiates RNAi- and heterochromatindependent gene silencing. Cell 125: 873 .

Carrington J.C. and Ambros V. 2003. Role of microRNAs in plant and animal development. Science 301: 336.

Chen C.C., Simard M.J., Tabara H., Brownell D.R., McCollough J.A., and Mello C.C. 2005. A member of the polymerase beta nucleotidyltransferase superfamily is required for RNA interference in C. elegans. Curr. Biol. 15: 378.

Dellino G.I., Schwartz Y.B., Farkas G., McCabe D., Elgin S.C., and Pirrotta V. 2004. Polycomb silencing blocks transcription initiation. Mol. Cell 13: 887.

Ekwall K., Nimmo E.R., Javerzat J.P., Borgstrom B., Egel R., Cranston G., and Allshire R. 1996. Mutations in the fission yeast silencing factors clr4+ and rik1+ disrupt the localisation 
of the chromo domain protein Swi6p and impair centromere function. J. Cell Sci. 109: 2637.

Eystathioy T., Chan E.K., Tenenbaum S.A., Keene J.D., Griffith K., and Fritzler M.J. 2002. A phosphorylated cytoplasmic autoantigen, GW182, associates with a unique population of human mRNAs within novel cytoplasmic speckles. Mol. Biol. Cell 13: 1338.

Filipowicz W., Jaskiewicz L., Kolb F.A., and Pillai R.S. 2005. Post-transcriptional gene silencing by siRNAs and miRNAs. Curr. Opin. Struct. Biol. 15: 331

Fire A., Xu S., Montgomery M.K., Kostas S.A., Driver S.E., and Mello C.C. 1998. Potent and specific genetic interference by double-stranded RNA in Caenorhabditis elegans. Nature 391: 806

Francis N.J. and Kingston R.E. 2001. Mechanisms of transcriptional memory. Nat. Rev. Mol. Cell Biol. 2: 409.

Grewal S.I. 2000. Transcriptional silencing in fission yeast. $J$. Cell. Physiol. 184: 311.

Grewal S.I. and Moazed D. 2003. Heterochromatin and epigenetic control of gene expression. Science 301: 798.

Grunstein M. 1997a. Histone acetylation in chromatin structure and transcription. Nature 389: 349.

- 1997b. Molecular model for telomeric heterochromatin in yeast. Curr. Opin. Cell Biol. 9: 383.

Hammond S.M., Bernstein E., Beach D., and Hannon G.J. 2000. An RNA-directed nuclease mediates post-transcriptional gene silencing in Drosophila cells. Nature 404: 293.

Hannon G.J. 2002. RNA interference. Nature 418: 244.

Haracska L., Johnson R.E., Prakash L., and Prakash S. 2005. Trf4 and Trf5 proteins of Saccharomyces cerevisiae exhibit poly(A) RNA polymerase activity but no DNA polymerase activity. Mol. Cell. Biol. 25: 10183.

Heitz E. 1928. Das heterochromatin der moose. Jahrb. Wiss. Botanik 69: 762 .

Henikoff S. 1998. Conspiracy of silence among repeated transgenes. Bioessays 20: 532 .

Hong E.-J.E., Villén J., Gerace E.L., Gygi S.P., and Moazed D. 2005. A Cullin E3 ubiquitin ligase complex associates with Rik1 and the Clr4 histone H3-K9 methyltransferase and is required for RNAi-mediated heterochromatin formation. $R N A$ Biol. 2: 106.

Horn P.J., Bastie J.N., and Peterson C.L. 2005. A Rik1-associated, cullin-dependent E3 ubiquitin ligase is essential for heterochromatin formation. Genes Dev. 19: 1705.

Hu J., McCall C.M., Ohta T., and Xiong Y. 2004. Targeted ubiquitination of CDT1 by the DDB1-CUL4A-ROC1 ligase in response to DNA damage. Nat. Cell Biol. 6: 1003.

Huttenhofer A., and Schattner P. 2006. The principles of guiding by RNA: Chimeric RNA-protein enzymes. Nat. Rev. Genet. 7: 475 .

Hutvagner G. and Zamore P.D. 2002. A microRNA in a multiple-turnover RNAi enzyme complex. Science 297: 2056.

Iida T., Kawaguchi R., and Nakayama J. 2006. Conserved ribonuclease, eri1, negatively regulates heterochromatin assembly in fission yeast. Curr. Biol. 16: 1459

Jenuwein T. and Allis C.D. 2001. Translating the histone code. Science 293: 1074

Jia S., Kobayashi R., and Grewal S.I. 2005. Ubiquitin ligase component $\mathrm{Cul} 4$ associates with Clr4 histone methyltransferase to assemble heterochromatin. Nat. Cell Biol. 7: 1007.

Justesen J., Hartmann R., and Kjeldgaard N.O. 2000. Gene structure and function of the $2^{\prime}-5^{\prime}$-oligoadenylate synthetase family. Cell. Mol. Life Sci. 57: 1593.

Kennedy S., Wang D., and Ruvkun G. 2004. A conserved siRNA-degrading RNase negatively regulates RNA interference in C. elegans. Nature 427: 645.

Kornberg R.D. and Lorch Y. 1999. Twenty-five years of the nucleosome, fundamental particle of the eukaryote chromosome. Cell 98: 285.

LaCava J., Houseley J., Saveanu C., Petfalski E., Thompson E., Jacquier A., and Tollervey D. 2005. RNA degradation by the exosome is promoted by a nuclear polyadenylation complex. Cell 121: 713 .
Lachner M., O'Carroll D., Rea S., Mechtler K., and Jenuwein T. 2001. Methylation of histone H3 lysine 9 creates a binding site for HP1 proteins. Nature 410: 116

Li F., Goto D.B., Zaratiegui M., Tang X., Martienssen R., and Cande W.Z. 2005. Two novel proteins, dos 1 and dos2, interact with rik1 to regulate heterochromatic RNA interference and histone modification. Curr. Biol. 15: 1448.

Lingel A., Simon B., Izaurralde E., and Sattler M. 2003. Structure and nucleic-acid binding of the Drosophila Argonaute 2 PAZ domain. Nature 426: 465.

Luger K., Mader A.W., Richmond R.K., Sargent D.F., and Richmond T.J. 1997. Crystal structure of the nucleosome core particle at 2.8 Å resolution. Nature 389: 251.

Makeyev E.V. and Bamford D.H. 2002. Cellular RNA-dependent RNA polymerase involved in posttranscriptional gene silencing has two distinct activity modes. Mol. Cell 10: 1417.

Martienssen R., Lippman Z., May B., Ronemus M., and Vaughn M. 2004. Transposons, tandem repeats, and the silencing of imprinted genes. Cold Spring Harbor Symp. Quant. Biol. 69: 371.

Meister G., Landthaler M., Patkaniowska A., Dorsett Y., Teng G., and Tuschl T. 2004. Human Argonaute2 mediates RNA cleavage targeted by miRNAs and siRNAs. Mol. Cell 15: 185 .

Moazed D. 2001. Common themes in mechanisms of gene silencing. Mol. Cell 8: 489.

Moazed D., Rudner A.D., Huang J., Hoppe G.J., and Tanny J.C. 2004. A model for step-wise assembly of heterochromatin in yeast. Novartis Found. Symp. 259: 48.

Mochizuki K., Fine N.A., Fujisawa T., and Gorovsky M.A. 2002. Analysis of a piwi-related gene implicates small RNAs in genome rearrangement in tetrahymena. Cell 110: 689.

Motamedi M.R., Verdel A., Colmenares S.U., Gerber S.A., Gygi S.P., and Moazed D. 2004. Two RNAi complexes, RITS and RDRC, physically interact and localize to noncoding centromeric RNAs. Cell 119: 789.

Nakayama J., Rice J.C., Strahl B.D., Allis C.D., and Grewal S.I. 2001. Role of histone H3 lysine 9 methylation in epigenetic control of heterochromatin assembly. Science 292: 110.

Noma K., Sugiyama T., Cam H., Verdel A., Zofall M., Jia S., Moazed D., and Grewal S.I. 2004. RITS acts in cis to promote RNA interference-mediated transcriptional and post-transcriptional silencing. Nat. Genet. 36: 1174.

Pal-Bhadra M., Bhadra U., and Birchler J.A. 2002. RNAi related mechanisms affect both transcriptional and posttranscriptional transgene silencing in Drosophila. Mol. Cell 9: 315 .

Pal-Bhadra M., Leibovitch B.A., Gandhi S.G., Rao M., Bhadra U., Birchler J.A., and Elgin S.C. 2004. Heterochromatic silencing and HP1 localization in Drosophila are dependent on the RNAi machinery. Science 303: 669.

Paro R. 1993. Mechanisms of heritable gene repression during development of Drosophila. Curr. Opin. Cell Biol. 5: 999.

Partridge J.F., Borgstrom B., and Allshire R.C. 2000. Distinct protein interaction domains and protein spreading in a complex centromere. Genes Dev. 14: 783.

Partridge J.F., Scott K.S., Bannister A.J., Kouzarides T., and Allshire R.C. 2002. cis-acting DNA from fission yeast centromeres mediates histone $\mathrm{H} 3$ methylation and recruitment of silencing factors and cohesin to an ectopic site. Curr. Biol. 12: 1652.

Pirrotta V. and Gross D.S. 2005. Epigenetic silencing mechanisms in budding yeast and fruit fly: Different paths, same destinations. Mol. Cell 18: 395 .

Plasterk R.H. and Ketting R.F. 2000. The silence of the genes. Curr. Opin. Genet. Dev. 10: 562.

Rea S., Eisenhaber F., O'Carroll D., Strahl B.D., Sun Z.W., Schmid M., Opravil S., Mechtler K., Ponting C.P., Allis C.D., and Jenuwein T. 2000. Regulation of chromatin structure by site-specific histone H3 methyltransferases. Nature 406: 593.

Read R.L., Martinho R.G., Wang S.W., Carr A.M., and Norbury C.J. 2002. Cytoplasmic poly(A) polymerases mediate cellular responses to S phase arrest. Proc. Natl. Acad. Sci. 99: 12079.

Reinhart B.J. and Bartel D.P. 2002. Small RNAs correspond to centromere heterochromatic repeats. Science 297: 1831. 


\section{RNAI AND HETEROCHROMATIN}

Richards E.J. and Elgin S.C. 2002. Epigenetic codes for heterochromatin formation and silencing: Rounding up the usual suspects. Cell 108: 489.

Rusche L.N., Kirchmaier A.L., and Rine J. 2003. The establishment, inheritance, and function of silenced chromatin in Saccharomyces cerevisiae. Annu. Rev. Biochem. 72: 481.

Sadaie M., Iida T., Urano T., and Nakayama J. 2004. A chromodomain protein, Chp1, is required for the establishment of heterochromatin in fission yeast. EMBO J. 23: 3825 .

Saitoh S., Chabes A., McDonald W.H., Thelander L., Yates J.R., and Russell P. 2002. Cid13 is a cytoplasmic poly(A) polymerase that regulates ribonucleotide reductase mRNA. Cell 109: 563 .

Song J.J., Liu J., Tolia N.H., Schneiderman J., Smith S.K., Martienssen R.A., Hannon G.J., and Joshua-Tor L. 2003. The crystal structure of the Argonaute2 PAZ domain reveals an RNA binding motif in RNAi effector complexes. Nat. Struct. Biol. 10: 1026 .

Sugiyama T., Cam H., Verdel A., Moazed D., and Grewal S.I. 2005. RNA-dependent RNA polymerase is an essential component of a self-enforcing loop coupling heterochromatin assembly to siRNA production. Proc. Natl. Acad. Sci. 102: 152

Thon G. and Verhein-Hansen J. 2000. Four chromo-domain proteins of Schizosaccharomyces pombe differentially repress transcription at various chromosomal locations. Genetics 155: 551.

Thon G., Hansen K.R., Altes S.P., Sidhu D., Singh G., Verhein-
Hansen J., Bonaduce M.J., and Klar A.J. 2005. The Clr7 and Clr8 directionality factors and the Pcu4 cullin mediate heterochromatin formation in the fission yeast Schizosaccharomyces pombe. Genetics 171: 1583.

Vanacova S., Wolf J., Martin G., Blank D., Dettwiler S., Friedlein A., Langen H., Keith G., and Keller W. 2005. A new yeast poly(A) polymerase complex involved in RNA quality control. PLoS Biol. 3: e189.

Verdel A. and Moazed D. 2005. RNAi-directed assembly of heterochromatin in fission yeast. FEBS Lett. 579: 5872.

Verdel A., Jia S., Gerber S., Sugiyama T., Gygi S., Grewal S.I., and Moazed D. 2004. RNAi-mediated targeting of heterochromatin by the RITS complex. Science 303: 672.

Volpe T.A., Kidner C., Hall I.M., Teng G., Grewal S.I., and Martienssen R.A. 2002. Regulation of heterochromatic silencing and histone H3 lysine-9 methylation by RNAi. Science 297: 1833

Wassenegger M., Heimes S., Riedel L., and Sanger H.L. 1994. RNA-directed de novo methylation of genomic sequences in plants. Cell 76: 567.

Yamada T., Fischle W., Sugiyama T., Allis C.D., and Grewal S.I. 2005. The nucleation and maintenance of heterochromatin by a histone deacetylase in fission yeast. Mol. Cell 20: 173.

Yan K.S., Yan S., Farooq A., Han A., Zeng L., and Zhou M.M. 2003. Structure and conserved RNA binding of the PAZ domain. Nature 426: 468. 


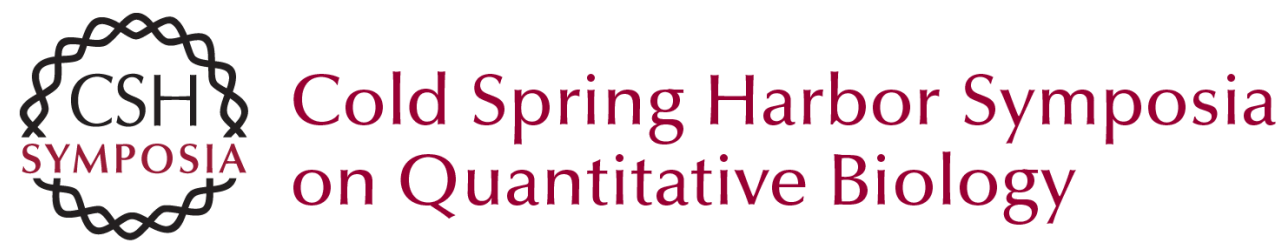

\section{Studies on the Mechanism of RNAi-dependent Heterochromatin Assembly}

D. MOAZED, M. BÜHLER, S.M. BUKER, et al.

Cold Spring Harb Symp Quant Biol 2006 71: 461-471

Access the most recent version at doi:10.1101/sqb.2006.71.044

References This article cites 75 articles, 22 of which can be accessed free at: http://symposium.cshlp.org/content/71/461.full.html\#ref-list-1

License

Email Alerting Receive free email alerts when new articles cite this article - sign up in Service the box at the top right corner of the article or click here. 